\title{
INSTANTANEOUS COASTLINE EXTRACTION FROM LIDAR POINT CLOUD AND HIGH RESOLUTION REMOTE SENSING IMAGERY
}

\author{
Li Yunfan ${ }^{1}$,Zhoing Liang ${ }^{1}$, Lai Zulong ${ }^{2}$,Gan Zheng ${ }^{1}$ \\ ${ }^{1}$ ChangJiang Spatial Infomation Technology Engineering Co.Ltd , Wuhan 430010 , China; \\ ${ }^{2}$ Faculty of Information Engineering, China University of Geosciences, Wu han 430074, China
}

\section{Commission III, WG III/5}

KEY WORDS: coastline, image segmentation, LiDAR, region growing

\begin{abstract}
:
A new method was proposed for instantaneous waterline extraction in this paper, which combines point cloud geometry features and image spectral characteristics of the coastal zone. The proposed method consists of follow steps:Mean Shift algorithm is used to segment the coastal zone of high resolution remote sensing images into small regions containing semantic information; Region features are extracted by integrating LiDAR data and the surface area of the image;initial waterlines are extracted by $\alpha$-shape algorithm.;a region growing algorithm with is taking into coastline refinement, with a growth rule integrating the intensity and topography of LiDAR data;moothing the coastline.Experiments are conducted to demonstrate the efficiency of the proposed method.
\end{abstract}

\section{INTRODUCTION}

Coastline extraction takes an important position in the investigation work of coastal zone. Coastline divides oceans and land administration baseline region is determined within the territorial sea boundary between water and land, but also the distinction between land and ocean depth reference elevation benchmarks boundary. Coastal zone plays an important role not only in ocean development, economic development, foreign trade, cultural exchange, etc., but also national security, military activities to the portal land. Coastal zone plays an important role not only in ocean development, economic development, foreign trade, cultural exchange, etc., but also national security, military activities gateway to land.

Current research mainly focuse only on the extraction of high-resolution satellite remote sensing images and synthetic aperture radar imaging(Niedermeier et al., 2000, $\mathrm{Du}$ et al., 2001).Airborne LiDAR, with ability to accurately express the three-dimensional topographic features, is a strong complement to remote sensing images. The current combination of LiDAR point cloud data and image edges Extraction of water far from mature, mostly from the point cloud derived nDSM as an added feature dimensions involved in terrain classification, or the use of highly accurate contour point cloud data, then get water and other contour lines(Chen et al., 2009, Wang et al., 2009). These methods have failed to fully exploit LiDAR geometric characteristics of the surface description capability.

A new method was proposed for instantaneous waterline extraction in this paper, which combines point cloud geometry features and image spectral characteristics of the coastal zone. The proposed method consists of follow steps:(1) Mean Shift algorithm is used to segment the coastal zone of high resolution remote sensing images into small regions containing semantic information.(2) Region features are extracted by integrating LiDAR data and the surface area of the image.(3) Initial waterlines are extracted by $\alpha$-shape algorithm.(4) A region growing algorithm with is taking into coastline refinement, with a growth rule integrating the intensity and topography of LiDAR data.(5)Smoothing the coastline.

\section{THE PROPOSED METHOD}

\subsection{Image Segmentation}

Mean Shift(Fukunaga et al., 1975) is a non-parametric kernel density estimation statistical iterative algorithm, it does not require any prior knowledge, and the data are valid for any 
distribution, the core of the sample point clustering feature space to obtain the modal point, namely the probability density is a local maximum value of the probability density gradient is zero points.

Mean Shift algorithm contains an iterative step, first calculate the mean deviation of the current point, move the shift points to their average, and then as a new starting point, continue to move until certain conditions are met over. Yizong Cheng of basic Mean Shift algorithm to do the following two aspects of the promotion, first define a family of kernel functions, such as the distance between different samples with the offset point, and its contribution to offset the mean shift vector is different It followed also sets a weighting factor, so that different points of importance is not the same sample, which greatly expanded the scope of the Mean Shift(Cheng, 1995). Comaniciu, who successfully applied the Mean Shift feature space analysis(Comaniciu et al., 2001), in the image smoothing and image segmentation Mean Shift have been widely used. Comaniciu the other evidence in the article, Mean Shift algorithm under certain conditions, will be able to converge to a steady-state point nearest the probability density function, therefore Mean Shift algorithm can be used to detect the presence of the probability density function modal.

\subsection{Water area extraction}

NDWI(McFeeters, 1996) similar normalized difference vegetation index NDVI, a Ratio Index, the basic principle is that in a multi-spectral bands, looking for the strongest and weakest reflection reflective bands bands to be studied to class, will put strong molecular weak placed denominator. By calculating the ratio, to further expand the gap between the two, then as normalized to unity between the numerical range $[-1,1]$, so that the feature of interest to get the maximum brightness of the image on the index generated enhanced, while others feature the background was generally suppressed.

Therefore, the use of LiDAR point cloud data on the expression of surface topological properties further filter error extracting water body, mainly by the following three features:

(1) Point cloud density difference

Since the specular reflection effect of the water, the density of water at the point cloud are often lower than on land. The assumption that all LiDAR point cloud data, where $\mathrm{N}$ is the total number of points, point contains the three-dimensional coordinates represented; is a point cloud data is divided into $\mathrm{M}$ blocks; means that all points within the i-th block. In the i-th block number as the number of point cloud point cloud is determined based on whether the sparse, that is, when a sparse representation, which is sparse point cloud judgment threshold.

(2)Elevation similarity degree

Compared with the land, the water close to the surface of the plane, elevation range of ups and downs in the local little change. The average elevation by elevation variance is determined whether the water body is similar. Block $\mathrm{i}$ is set to the mean square height, if, the block is a horizontal plane, which is to determine whether a threshold level.

(3)Echo strength calculation

Currently, most of the airborne LiDAR system uses near-infrared laser, has a strong absorption effect in the body of water, and are therefore the point cloud water at the echo intensity is generally low. Typically, playing in the water echo intensity laser spot weak, and strong play in the water utility point of echo intensity, echo intensity in order to obtain the true body of water, you must first remove the debris above the water point, and then statistical playing in the water point average echo intensity as echo intensity in the region. If the area is less than the strength of the echo, it indicates that the weak echo region.

\subsection{Initial waterlines extraction}

Firstly previously extracted mainly sea water on the water point cloud classification mark. Point cloud data for each laser pin point iteration, using the formula (1) to $\mathrm{X}, \mathrm{Y}$ coordinates are transformed into the image coordinates, and then extracted from sea water corresponding result image pixels determine whether the 
waters, if so, the laser marker pin point water point, thus completing the water point cloud marker.

$$
\begin{gathered}
X_{\text {img }}=\operatorname{int}\left(X-X_{\min } / \text { Pixelsize }\right) \\
Y_{\text {img }}=\operatorname{int}\left(Y-Y_{\min } / \text { Pixelsize }\right)
\end{gathered}
$$

After analysis of many of the existing literature, we use a two-dimensional $\alpha$-shapes algorithm for point cloud water body contour extraction(Edelsbrunner et al., 1983). A finite set of points $S$ of $\alpha$-shape is a $\alpha$ by a set of points $\mathrm{S}$ and parameters uniquely determined polygons, intuitive performance of the point set $S$ shape parameter $\alpha$ controls the level of granularity polygon generation of, and may process concave polygon boundary.

Advantages of $\alpha$-shapes algorithm: (1) $\alpha$-shapes previously constructed Delaunay triangulation, so each triangle of three vertices are arranged in counter-clockwise order, each edge of the triangle is actually a vector from the start point to the end point. Therefore, when we get to all of the $\alpha$-shapes side by side to connect these vectors end to end configuration, you can get a sequence of vertices counterclockwise arrangement, resulting in a complete point cloud water body contour polygons.(2) $\alpha$-shapes algorithm parameters simple algorithm in favor of automation. Shen Wei pointed out that, under normal circumstances, $\alpha$ is set at the average point spacing is more suitable between 1 to 2 times(Shen et al., 2008). (3) $\alpha$-shapes algorithm can extract only the outer contour point sets, but also can extract the outline of the internal cavity, it is conducive to acquiring and processing island (reef) and other closed coastline.

\subsection{Coastline refinement}

Near the natural shoreline, the waves hit the rocks produce a lot of spray, the main body of water waters exhibit distinct spectral characteristics, its normalized water index tend to be between the sea and the land, it is difficult to be extracted directly from the image. At the same time, due to the presence of rocks, with waves in the LiDAR point cloud data exhibit high roughness, showed some of the characteristics of the land. It features with the full application of the waves on the surface texture of the spectrum and can be extracted.

Extracted by the above method showing a spot with the waves, but due to image segmentation is not considered terrain, many point cloud polygon (especially with the land adjacent polygons) are not included in the surf zone entirely, so by the waves with a result of the extraction method can not be directly used as the final waterlines. Coastline near the point cloud to do a cross-sectional analysis shows that the vertical direction. The surface of the water near the coastline of point cloud Despite the surging waves of the shore to produce a slight slope, but with the land boundary line Department still has a more obvious differences in slope can be used as a basis for extraction; at the same time, the relatively flat, it is difficult to distinguish between changes in the slope of the coastline, adding strength to constrain feature point cloud, a comprehensive two, you can get the nearest land and sea boundary position . Based on this, this paper presents a point cloud into account the terrain and intensity region growing algorithm, described as follows:

\subsection{Coastline smoothing}

Through the above process to give a precise spatial location of the instantaneous water edge, but as a practical application of geographic features, such a detail waterlines too much detail, contains more burrs and the inflection point, need to be smooth. Curve smoothing is required between the apex initial curve vector contained in the interpolation, such as B-spline function(Ligun et al., 1998), cubic spline function or tension spline function, etc., is common in the like high line optimization process[4]. Tension Spline interpolation method has a good effect, therefore, this paper tension spline function interpolation for smooth conduct waterlines.

\section{EXPERIMENT}

\subsection{Dataset}

To verify the effectiveness of the method, we selected a group of typical coastal area of experimental data (Figure 1), including loading LiDAR point cloud, aerial imagery and near-infrared images. Point cloud which contains the 3.3 million laser foot points collected by the Optech ALTM 3100EA system, the average point spacing of $0.7 \mathrm{~m}$, the average point density on land ranging from about 2.18 points / $\mathrm{m} 2$; average point spacing in the ocean range from 1.5 points / $\mathrm{m} 2$. Figure 1 (a) for the elevation of the rendering mode point cloud. Test region imagary (Figure 1(b)) and near-infrared imagary(Figure 
1(c)) collected by the Applanix DSS (Digital Sensor

System) Aviation camera acquisition, spatial resolution of $0.5 \mathrm{~m}$, have been orthorectification process.

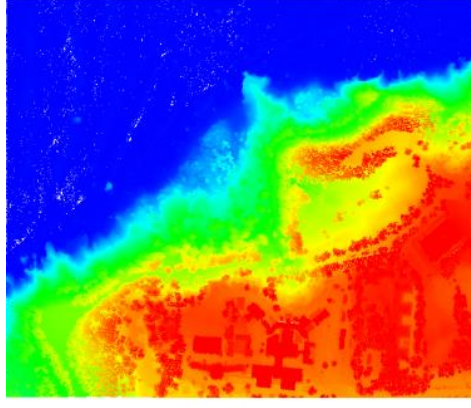

(a)

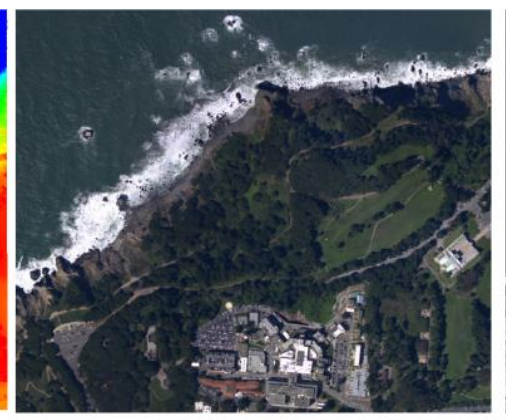

(b)

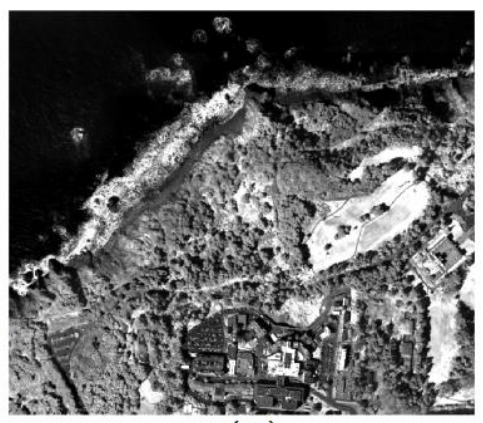

(c)

Figure 1. Experiment dataset.

(a)LiDAR point cloud in elevation rendering mode;(b)aerial imagery;(c)near-infrared imagary.

\subsection{Water area extraction}

Experimental use of true color image area and near infrared imaging, according to the formula (3-13) calculated NDWI raster data, Figure 3-22 for the calculation results can be seen from the water and land with a large difference in brightness. Polygon ranges are calculated based on the object image obtained by dividing the mean of each polygon NDWI internal and select NDWI> 0.55 as the extraction threshold value of the initial body of water extraction result shown in Figure 3-23. Through observation, can be found in the main body of water extracted is substantially correct, as shown in Figure 3-24. But it also has a lot of shades are mistaken as a body of water, resulting in errors.

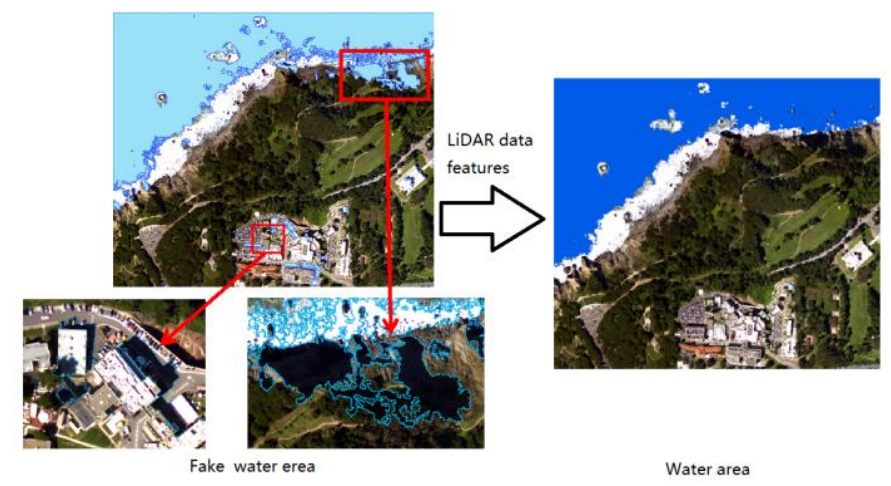

Figure 2. result of water area extraction integrating

NDWI and LiDAR data features

\subsection{Coastline extraction}

The initial results of the use of sea water extract obtained above, point cloud category tags, then a two-dimensional $\alpha$-shapes algorithm to give its outline (Figure 3), wherein the $\alpha$ parameter binding average point spacing consider this article experimental data using $1.5 \mathrm{~m}$. The results can be seen that $\alpha$-shapes can complete extract is marked as point cloud sea water inside and outside contour. Among them, a small amount of reefs will produce voids, can be more easily removed through the area threshold.
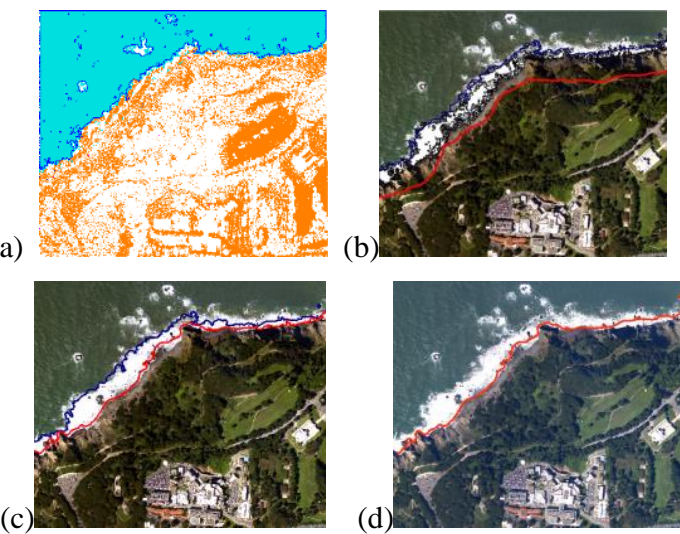

Figure 3. Results of the coastline extraction.

(a)result of water area extraction;(b)result of intial waterlines extraction;(c)result of coastline refinement;(d)result of coastline smoothing

\section{CONCLUSION}

Extraction coastline plays an important role in coastal survey information, instantaneous water extracted edges by means of remote sensing coastline extraction is a 
necessary step. For the present research, this paper presents waterlines extraction method combined with LiDAR point clouds and Imagery: (1) First images for Mean Shift segmentation algorithm, and using the normalized difference water index extracted from the initial waters water body polygons; the waves (2) after the two-dimensional $\alpha$-shapes algorithm initial water boundaries, proposed to take into account the strength of point clouds and terrain features triangle growing algorithm, and surface characteristics of a spectral band was more complex extraction, and generates the waterlines after refinement; (3) Finally, the tension spline function method waterlines were smooth. It proved the effectiveness of the proposed method in this paper.

\section{REFERENCES}

Chen, Y., W. Su, J. Li and Z. Sun,2009. Hierarchical object oriented classification using very high resolution imagery and LIDAR data over urban areas. Advances in Space Research 43(7): 1101-1110.

Cheng, Y.,1995. Mean shift, mode seeking, and clustering. Pattern Analysis and Machine Intelligence, IEEE Transactions on 17(8): 790-799.

Comaniciu, D., V. Ramesh and P. Meer,2001. The variable bandwidth mean shift and data-driven scale selection. Computer Vision, 2001. ICCV 2001. Proceedings. Eighth IEEE International Conference on, IEEE.

Du, J.-K., Y.-S. Huang, X.-Z. Feng and Z.-1. Wang,2001. Study on water bodies extraction and classification from SPOT image. JOURNAL OF REMOTE SENSING-BEIJING- 5(3): 219-225.

Edelsbrunner, H., D. Kirkpatrick and R. Seidel,1983. On the shape of a set of points in the plane. Information Theory, IEEE Transactions on 29(4): 551-559.

Fukunaga, K. and L. Hostetler,1975. The estimation of the gradient of a density function, with applications in pattern recognition. Information Theory, IEEE Transactions on 21(1): $32-40$

Ligun, A., A. Shumeiko, S. P. Radzevich and E. D. Goodman,1998. Asymptotically optimum recovery of smooth contours by Bezier curve. Computer aided geometric design 15(5): 495-506.
McFeeters, S.,1996. The use of the Normalized Difference Water Index (NDWI) in the delineation of open water features. International journal of remote sensing 17(7): 1425-1432.

Niedermeier, A., E. Romaneessen and S. Lehner,2000. Detection of coastlines in SAR images using wavelet methods. IEEE Transactions on Geoscience and Remote Sensing 38(5): 2270-2281.

Shen, W., J. Li, Y.-h. Chen, L. Deng and G.-x. Peng,2008. Algorithms study of building boundary extraction and normalization based on Lidar data. Journal of Remote Sensing 5: 003.

Wang, Z., H. MA and H. XU,2009. Approach for water body extraction combing point clouds with image. Comput. Eng. Appl 45(21): 33-36. 